\title{
Vector Potential Remap for 2d MHD
}

\author{
P. W. Rambo
}

October 13, 1998

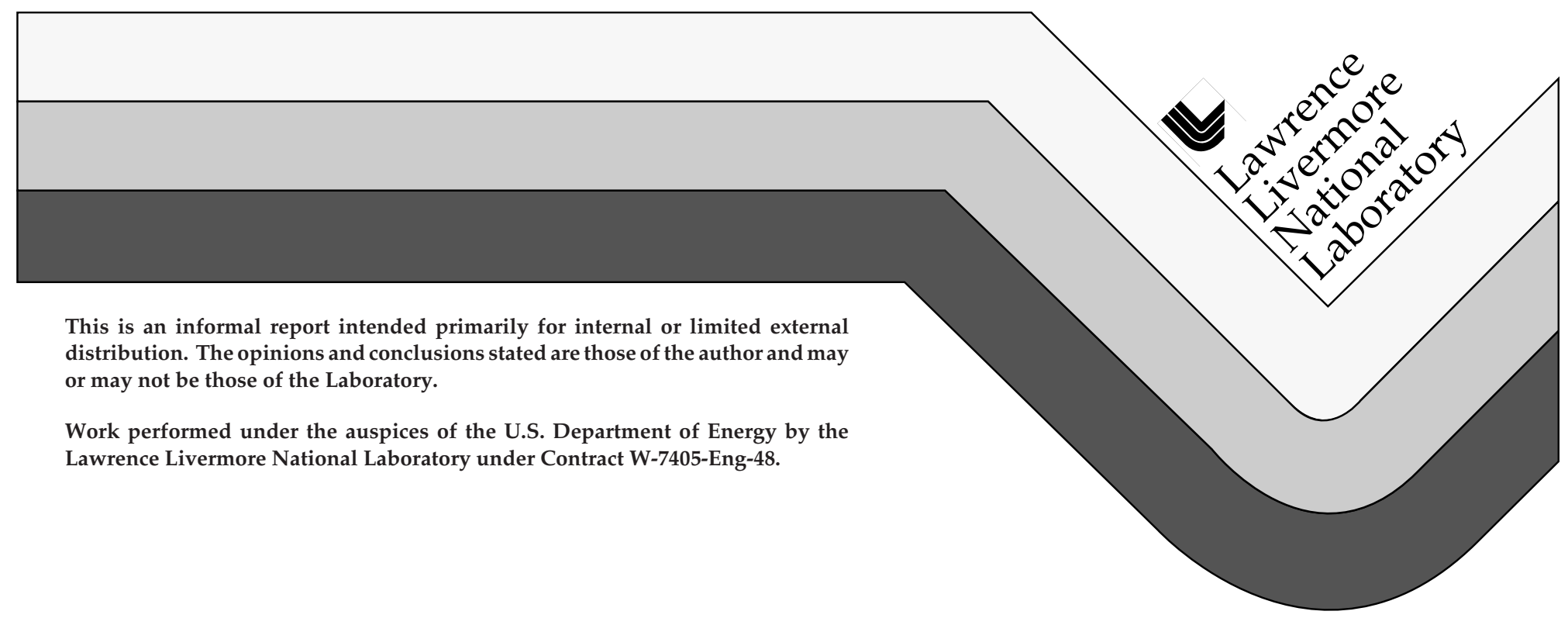




\section{DISCLAIMER}

This document was prepared as an account of work sponsored by an agency of the United States Government. Neither the United States Government nor the University of California nor any of their employees, makes any warranty, express or implied, or assumes any legal liability or responsibility for the accuracy, completeness, or usefulness of any information, apparatus, product, or process disclosed, or represents that its use would not infringe privately owned rights. Reference herein to any specific commercial product, process, or service by trade name, trademark, manufacturer, or otherwise, does not necessarily constitute or imply its endorsement, recommendation, or favoring by the United States Government or the University of California. The views and opinions of authors expressed herein do not necessarily state or reflect those of the United States Government or the University of California, and shall not be used for advertising or product endorsement purposes.

This report has been reproduced directly from the best available copy.

Available to DOE and DOE contractors from the Office of Scientific and Technical Information P.O. Box 62, Oak Ridge, TN 37831

Prices available from (423) 576-8401

Available to the public from the National Technical Information Service

U.S. Department of Commerce 5285 Port Royal Rd. Springfield, VA 22161 


\title{
Vector Potential Remap for 2d MHD*
}

\author{
Peter W. Rambo \\ Lawrence Livermore National Laboratory
}

This report describes an algorithm to remap (rezone) the magnetic vector potential in 2D MHD calculations. This extends a previously described Lagrangian scheme [1] for use with ALE grids, when combined with a suitable remap capability for the hydrodynamic variables. In this scheme, the magnetic field $\mathbf{B}$ is a zone quantity derived from the node based vector potential $\mathbf{A}\left(B_{\mathrm{x}}, B_{\mathrm{y}}\right.$, and $A_{\mathrm{z}}$ in Cartesian geometry, $B_{\mathrm{z}}, B_{\mathrm{r}}$, and $A_{\phi}$ in cylindrical geometry). The scheme outlined here is closely related to a flux conserving remap of the magnetic field. Because $\mathbf{B}$ is derived from the updated vector potential, however, flux conservation and vanishing divergence are guaranteed; only accuracy is a concern. This is in contrast to schemes based directly on $\mathbf{B}$, which require the additional constraint of preserving zero divergence $[2,3]$. Beginning with Cartesian geometry, a straight forward development of the algorithm is first presented; an alternative view is then considered which makes clear the relationship to a flux conservative remapping of the zonal magnetic field. Examples comparing first and second order algorithms are shown, and the issue of energy conservation is discussed. Finally, the generalization to cylindrical geometry is given.

Figure 1 shows a diagram of a single quadrilateral zone, or cell, in the computational mesh defined by the surrounding node locations $\mathbf{r}_{\mathrm{n}}$. In Cartesian geometry, the zonal magnetic field, $\mathbf{B}_{\mathrm{c}}$, is determined from the $z$-component of the nodal vector potential, $A_{\mathrm{n}}$. In particular, we wish to know how to change $A_{\mathrm{n}}{ }^{0}$ to $A_{\mathrm{n}}{ }^{\mathrm{t}}$ due to moving a node $\delta \mathbf{r}_{\mathrm{n}} \equiv \mathbf{r}_{\mathrm{n}}{ }^{\mathrm{t}}-\mathbf{r}_{\mathrm{n}}{ }^{0}$, where superscripts " 0 " and " $t$ " will denote the original and remapped quantities respectively. We consider a surface perpendicular to the simulation plane with normal directed area $\delta \mathbf{S}=\delta \mathbf{r}_{\mathrm{n}} \times \hat{\mathbf{z}}$. Then the magnetic flux through this surface is related to the line integral of the vector potential,

$$
\int \mathbf{B} \cdot \mathbf{d S}=\int \nabla \times \mathbf{A} \cdot \mathbf{d} \mathbf{S}=\oint \mathbf{A} \cdot \mathbf{d l} \mathbf{l} .
$$

Applying this to the surface defined above,

$$
\delta A_{n} \equiv A_{n}^{t}-A_{n}^{0}=\hat{\mathbf{z}} \cdot \int_{\mathbf{r}_{n}^{0}}^{\mathbf{r}_{n}^{t}} \mathbf{B}(\mathbf{r}) \times \mathbf{d r} .
$$

Thus we need only calculate the integral of the magnetic flux to determine the change in the vector potential. Assume that within the cell, $\mathbf{B}(\mathbf{r})$ has linear variation about the average value relative to the cell centroid $\mathbf{r}_{\mathrm{c}}$,

$$
\mathbf{B}(\mathbf{r})=\mathbf{B}_{c}^{0}+\hat{\mathbf{x}}\left(\mathbf{r}-\mathbf{r}_{c}\right) \cdot\left(\nabla B_{x}\right)_{c}+\hat{\mathbf{y}}\left(\mathbf{r}-\mathbf{r}_{c}\right) \cdot\left(\nabla B_{y}\right)_{c} .
$$

The gradient terms within a cell are determined by suitable approximation using surrounding cell values, and will be discussed later. Substituting Eq. (3) into Eq. (2) and performing the integration,

$$
\begin{aligned}
\delta A_{n} \hat{\mathbf{z}} & =\left\{\mathbf{B}_{c}^{0}+\hat{\mathbf{x}}\left(\overline{\mathbf{r}}_{n}-\mathbf{r}_{c}\right) \cdot\left(\nabla B_{x}\right)_{c}+\hat{\mathbf{y}}\left(\overline{\mathbf{r}}_{n}-\mathbf{r}_{c}\right) \cdot\left(\nabla B_{y}\right)_{c}\right\} \times \delta \mathbf{r}_{n} \\
& \equiv \mathbf{B}_{n}^{A} \times \delta \mathbf{r}_{n} ; \quad \mathbf{B}_{n}^{A}=\mathbf{B}\left(\mathbf{r}=\overline{\mathbf{r}}_{n}\right), \quad \overline{\mathbf{r}}_{n} \equiv \frac{1}{2}\left(\mathbf{r}_{n}^{0}+\mathbf{r}_{n}^{t}\right)=\mathbf{r}_{n}^{0}+\frac{1}{2} \delta \mathbf{r}_{n} .
\end{aligned}
$$


This defines an effective node magnetic field associated with the remap that is simply Eq. (3) evaluated at the intermediate node position. This is a second order algorithm, with a first order upwind scheme defined by neglecting the gradient terms. The new magnetic field is then determined from the updated mesh and vector potential; in Cartesian geometry this is given by,

$$
\mathbf{B}_{c} V_{c} \equiv \sum_{n}^{c} \mathbf{r}_{n} \Delta A^{n, c}, \quad \Delta A^{n, c} \equiv \frac{1}{2}\left(A_{n+1}-A_{n-1}\right),
$$

with all quantities evaluated at the new time level.

This remap is closely related to a flux conserving rezone of the magnetic field. If one specializes to one dimension, say with $B_{\mathrm{y}}$ varying in $x$, the (transverse) magnetic field is simply a zone defined scalar. Then an exact correspondence exists between this scheme and a conservative rezone of $B_{\mathrm{y}}$. Eq. (4) with the gradient terms set to zero corresponds exactly to donor cell advection; similarly, Eq. (4) with appropriately limited gradients is equivalent to a monotonic second order advection. In two dimensions, the vector nature of B complicates this, but an interesting correspondence still exists. Dukowicz and Kodis [4], and Ramshaw [5] have developed efficient methods for conservative rezoning of zonal scalar quantities on a quadrilateral mesh. This is based on defining the zonal quantity as the divergence of a vector field; then the volume overlaps between the old mesh and an arbitrary new mesh may be calculated as surface integrals. An analogous method for a flux conserving rezone of the magnetic field is possible by defining the vector potential in each cell of the old mesh as,

$$
A(\mathbf{r}) \hat{\mathbf{z}}=\mathbf{B}_{c}^{0} \times \mathbf{r}+\frac{1}{2}\left\{\left(\mathbf{r}-\mathbf{r}_{c}\right) \cdot(\nabla \mathbf{B})_{c}\right\} \times\left(\mathbf{r}-\mathbf{r}_{c}\right) ;
$$

the curl of Eq. (6) is exactly Eq. (3). Now a conservative rezone of $\mathbf{B}$ could be accomplished by calculating overlap integrals between the new and old mesh as surface integrals over $A$. Because the vector potential prescribed above is not continuous from one zone to an adjoining zone, care must be taken in accounting for delta-function contributions at old zone edges, exactly as prescribed in Ref. [5]. This rezone would not necessarily preserve zero divergence, so a divergence clean would then be necessary. The present scheme may be regarded as preparing to do a conservative remap in the same fashion, but instead of calculating volume overlaps of $\mathbf{B}$, the zone defined vector potential, Eq. (6), is evaluated at the new node location and from this a new divergence-free magnetic field is calculated. Because of possible discontinuities across zone boundaries, the change in $A_{\mathrm{n}}$ is calculated; taking the difference between Eq. (6) evaluated at both new and old node positions is exactly the same as Eq. (4).

Because the magnetic field calculated from the new vector potential is guaranteed to be divergence free, no effort seems necessary to ensure that the linear magnetic field of Eq. (3) is similarly solenoidal. Thus all four gradient components are limited independently and $-\left(\partial B_{\mathrm{x}} / \partial x\right)_{\mathrm{c}} \neq\left(\partial B_{\mathrm{y}} / \partial y\right)_{\mathrm{c}}$. Dukowicz and Kodis [4] describe methods for approximating these limited gradients based on the values of the gradients at the four nodes surrounding a zone. Finally, although presented here as though the node position were being moved an incremental distance, this is not necessary; the new mesh may be entirely unrelated to the old mesh. All that is required for application of the algorithm is to determine which old zone contains the new node position, and which old node is in closest proximity.

Several examples are now presented to demonstrate this remap algorithm. The advection of a square pulse on a fixed grid in one dimension is a standard test; Fig. 2 shows results of advecting an initially square $\mathrm{B}_{\mathrm{y}}$ profile in the $\mathrm{x}$-direction for $200 \Delta \mathrm{t}$, with a Courant number $\mathrm{u}_{0} \Delta \mathrm{t} / \Delta \mathrm{x}=0.2$. The solid line shows results from the second order algorithm, Eq. (4), with the field gradients limited using the monotonic limiter described in 
Ref. [4]; similar results were also obtained using the van Leer limiter. The dashed line shows results for the first order remap given by neglecting field gradients, which is seen to be very diffusive. As previously discussed, this remap in one dimension is exactly equivalent to a conservative rezone of $\mathrm{B}_{\mathrm{y}}$; the results in Fig. 2 clearly resemble typical results for conservative advection schemes. This remap algorithm is also equivalent to an advection $\left(\delta \mathbf{r}_{\mathrm{n}} \equiv \mathbf{u}_{\mathrm{n}} \delta \mathrm{t}\right)$ of the vector potential with a particular definition for the gradient,

$$
A_{n}^{t}=A_{n}^{0}+\delta t \mathbf{u}_{n} \cdot(\nabla A)_{n} \quad, \quad(\nabla A)_{n} \equiv \hat{\mathbf{z}} \times \mathbf{B}_{n}^{A} .
$$

This choice ensures monotonicity (at least in one dimension), and thus removes this criticism of vector potential schemes for MHD [2].

Although magnetic flux conservation is guaranteed, energy conservation is not. For the square wave advection test above, the field energy has decreased $23 \%$ for the low order remap, and $16 \%$ for the second order remap. The change in energy caused by the remap may be investigated by linearizing the change in field energy of the four zones surrounding a node that is being moved. There are two contributions: the first contribution from node displacement (constant potential) is related to the energy conserving node force [1]; the second contribution comes from the change in potential, and may be recognized as an electric field dotted with the node current, $\mathbf{I}_{\mathrm{n}}$. Defining a node magnetic field associated with the $\mathbf{J} \times \mathbf{B}$ force, as well as the one associated with the advection,

$$
\mathbf{F}_{n} \equiv \mathbf{I}_{n} \times \mathbf{B}_{n}^{F} / c, \text { and } \delta A_{n} \equiv \hat{\mathbf{z}} \cdot\left(\mathbf{B}_{n}^{A} \times \delta \mathbf{r}_{n}\right),
$$

the remap change in energy associated with a node displacement can be written as,

$$
\delta \varepsilon_{n}=-\mathbf{I}_{n} \times\left(\mathbf{B}_{n}^{F}-\mathbf{B}_{n}^{A}\right) \cdot \delta \mathbf{r}_{n} / c
$$

This change in energy is not necessarily dissipative; simple examples may be constructed which have positive energy change. An energy conserving remap is readily apparent by choosing the advection magnetic field equal to the field associated with the force, but stability would be sacrificed because of the loss of upwinding. Alternatively, one might adjust the fluid internal energy for conservation; this could be done through Eq. (9), or by advecting the sum of internal energy and field energy, then subtracting the field energy determined by the flux remap to find new internal energy. Because the remap is not guaranteed to be dissipative, however, this seems a risky proposition. Clearly the energy change due to the remap should be monitored to ensure it is properly small. Interestingly, since the magnitude of this energy change depends on the lack of spatial centering, use of the purely upwind field $\mathbf{B}_{\mathrm{c}}$ can aggravate the rate of energy increase, as well as amplify the rate of dissipation compared to a second order algorithm.

As a non-trivial two dimensional example, results are now presented for a magnetized Kelvin Helmholtz instability, a problem investigated by Malagoli et al. [6]. In their work, the unstable magnetized shear flow is simulated through nonlinear saturation using a highorder Godunov MHD code. Here, the identical case is simulated using a PIC-MHD code; in this code the fluid equations are solved using particles to advect the hydrodynamic information through the grid [7]. Unlike Brackbill's FLIP-MHD code [8], however, the PIC particles represent only the hydrodynamics, while the magnetic field is purely a grid quantity, advanced during the Lagrangian step as described in Ref. [1] and remapped to the original square grid (mesh spacing $\Delta$ ) as prescribed here. Because of the energy conserving properties of the PIC hydrodynamics, essentially the only dissipation is from Ohmic heating (diffusion coefficient $\mathrm{D}_{\mathrm{m}}$ ) and the field remap. The system is initially prepared with the fluid density uniform in space $\left(\rho_{0}\right)$, as is the field which is initially aligned with the $x$ direction; a shear flow is initialized with $\mathrm{u}_{\mathrm{x}}=\mathrm{u}_{0} \tanh (\mathrm{y} / \mathrm{a})$. The simulation region is $0<\mathrm{x}<\mathrm{L}$ 
and $-\mathrm{L}<\mathrm{y}<\mathrm{L}$ with periodic boundary conditions in $\mathrm{x}$ and reflecting walls at $\mathrm{y}= \pm \mathrm{L}$. For the case to be presented here the shear gradient parameter $\mathrm{a} / \mathrm{L}=0.05$, and $\mathrm{u}_{0} / \mathrm{C}_{\mathrm{s}}=0.5, \mathrm{C}_{\mathrm{s}}$ the adiabatic sound speed; the initial field strength is parameterized by the Alfven speed, $\mathrm{V}_{\mathrm{a}} / \mathrm{C}_{\mathrm{s}}=10^{-3}$. The fluid velocity is initially perturbed in the y-direction at the level of a few percent, with wavelength equal to the system length. Figures 3 and 4 show results from simulations with $\mathrm{L} / \Delta=100$, and a magnetic Reynolds number $\mathrm{Rm} \equiv \mathrm{Lu}_{0} / \mathrm{D}_{\mathrm{m}}=1000$. The remap of the vector potential uses Van Leer limiting. Contours of constant vector potential are shown in Fig. 3 at times corresponding to peak field energy, $\mathrm{C}_{\mathrm{s}} \mathrm{t} / \mathrm{L}=5.0$, and after saturation and reconnection, $\mathrm{C}_{\mathrm{s}} \mathrm{t} / \mathrm{L}=8.0$. The time history of the magnetic field energy is shown in Fig 4, as well as the rate of energy dissipation due to the field remap, which shows for a brief period of time near peak amplitude that the change in field energy due to the remap is positive, rather than dissipative. For these parameters, the total change in energy due to the remap is less than $40 \%$ of the initial field energy; the energy dissipated due to Ohmic heating is approximately ten times greater. Also shown in Fig. 4 are results from an otherwise identical simulation, but using the first order remap. As can be seen by comparing the rate of energy change due to remapping for the two cases, the rate of dissipation and energy increase are both accentuated by the low order scheme. The time and peak value of the magnetic energy compare well with the results of Malagoli, although it should be stressed that in the simulations of Ref. [6], no explicit field diffusion is included and the dissipation is only due to numerical causes.

This scheme may be applied to axisymmetric simulation by generalizing the integration that leads to Eq. (4). Now the integration is over a surface of revolution about the cylindrical axis, introducing factors of $2 \pi \mathrm{r}$ in both the surface and line integrals,

$$
\begin{aligned}
\delta \psi_{n} \equiv\left(r_{n}^{t} A_{n}^{t}\right)-\left(r_{n}^{0} A_{n}^{0}\right)=\bar{r}_{n}\left(\mathbf{B}_{c}^{0} \times \delta \mathbf{r}_{n}\right) \cdot \hat{\varphi} \\
+\delta r_{n}\left[\left\{\bar{r}_{n}\left(\bar{z}_{n}-z_{c}\right)+\frac{\delta r_{n} \delta z_{n}}{12}\right\}\left(\frac{\partial B_{z}}{\partial z}\right)_{c}+\left\{\bar{r}_{n}\left(\bar{r}_{n}-r_{c}\right)+\frac{\left(\delta r_{n}\right)^{2}}{12}\right\}\left(\frac{\partial B_{z}}{\partial r}\right)_{c}\right] \\
-\delta z_{n}\left[\left\{\bar{r}_{n}\left(\bar{z}_{n}-z_{c}\right)+\frac{\delta r_{n} \delta z_{n}}{12}\right\}\left(\frac{\partial B_{r}}{\partial z}\right)_{c}+\left\{\bar{r}_{n}\left(\bar{r}_{n}-r_{c}\right)+\frac{\left(\delta r_{n}\right)^{2}}{12}\right\}\left(\frac{\partial B_{r}}{\partial r}\right)_{c}\right] .
\end{aligned}
$$

The first term is the zero order result, followed by corrections for second order.

* This work was performed under the auspices of the U.S. Department of Energy by the Lawrence Livermore National Laboratory under Contract No. W-7405-Eng-48.

[1] P. W. Rambo, "Spatial Differencing of Poloidal MHD for Axisymmetric Lagrangian Simulation," Proceedings of the 16th Int. Conf. on the Numerical Simulation of Plasmas, Santa Barbara CA (Feb. 10-12, 1998).

[2] Charles R. Evans and John F. Hawley, Astrophys. J. 332, 659 (1988).

[3] Robert E. Peterkin Jr., Michael H. Frese, and Carl R. Sovinec, J. Comput. Phys. 140, 148 (1998).

[4] John K. Dukowicz and John W. Kodis, SIAM J. Sci. Stat. Comput. 8, 8 (1987).

[5] John D. Ramshaw, J. Comput. Phys. 67, 214 (1986).

[6] Andrea Malagoli, Gianluigi Bodo, and Robert Rosner, Astrophys. J. 456, 708 (1996).

[7] J. U. Brackbill and H. M. Ruppel, J. Comput. Phys. 65, 314 (1986).

[8] J.U. Brackbill, J. Comput. Phys. 96, 163 (1991). 

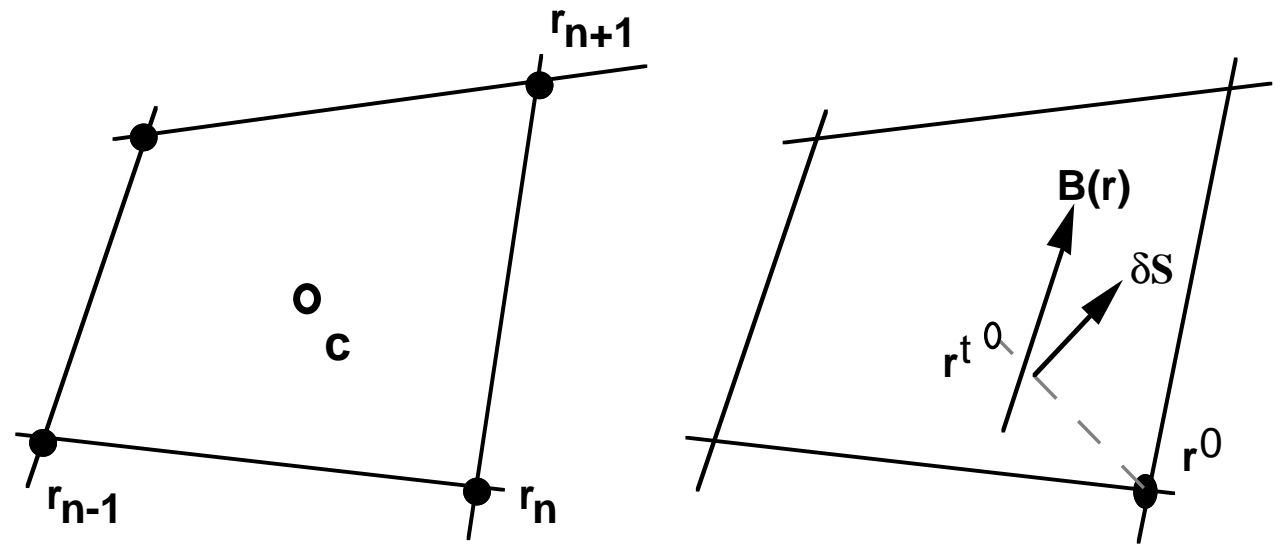

Fig. 1 Diagram showing quadrilateral zone defined by node vectors (left), and geometry of surface integral (right) for displacement of node-n into zone-c.

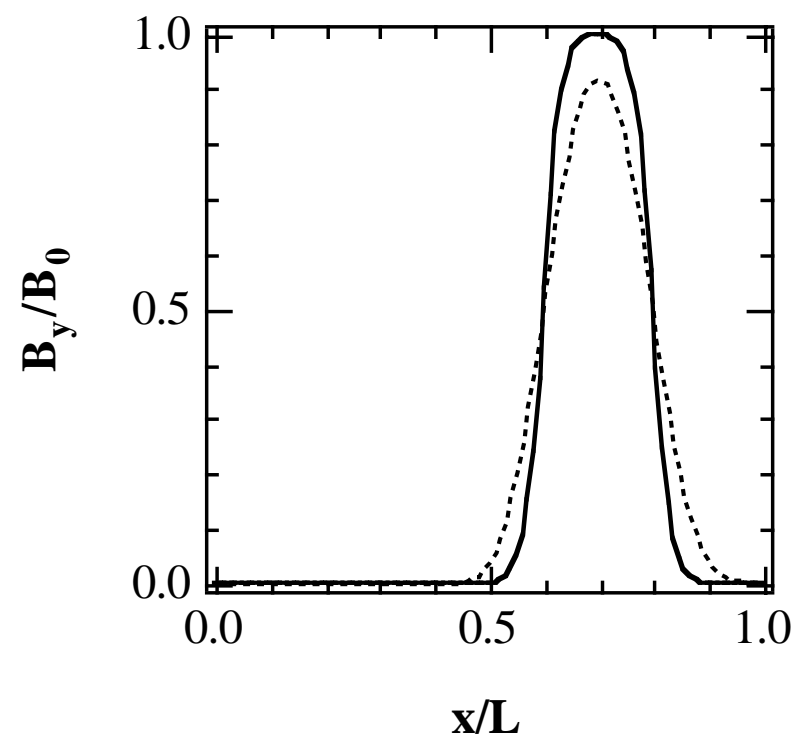

Fig. 2 One dimensional advection test at time $\mathrm{t}=200 \Delta \mathrm{t}=0.4 \mathrm{~L} / \mathrm{u}_{0}$; solid line is result with second order monotonic limited remap and dashed line shows result from first order remap; initial profile was square wave with width of $0.2 \mathrm{~L}=20 \Delta \mathrm{x}$. 

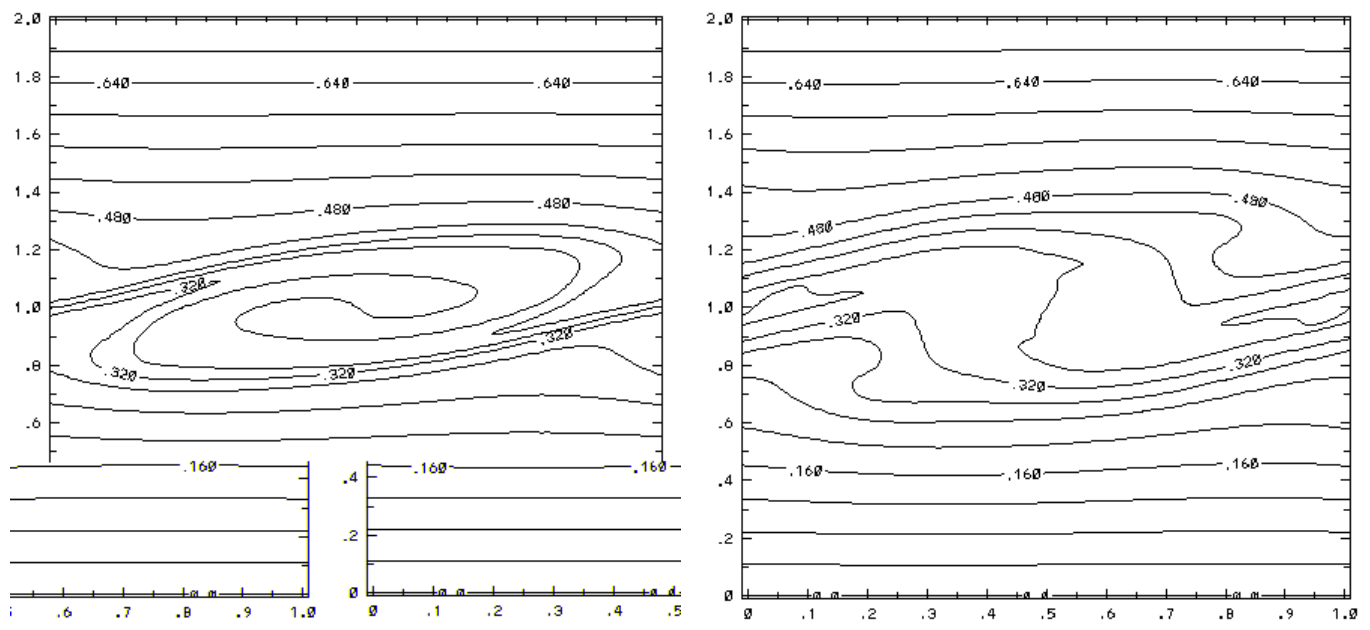

Fig. 3 Contours of constant vector potential at time near peak field magnitude, and well after saturation $\left(\mathrm{C}_{\mathrm{s}} \mathrm{t} / \mathrm{L}=5.0\right.$ left, and $\mathrm{C}_{\mathrm{s}} \mathrm{t} / \mathrm{L}=8.0$ right $)$.
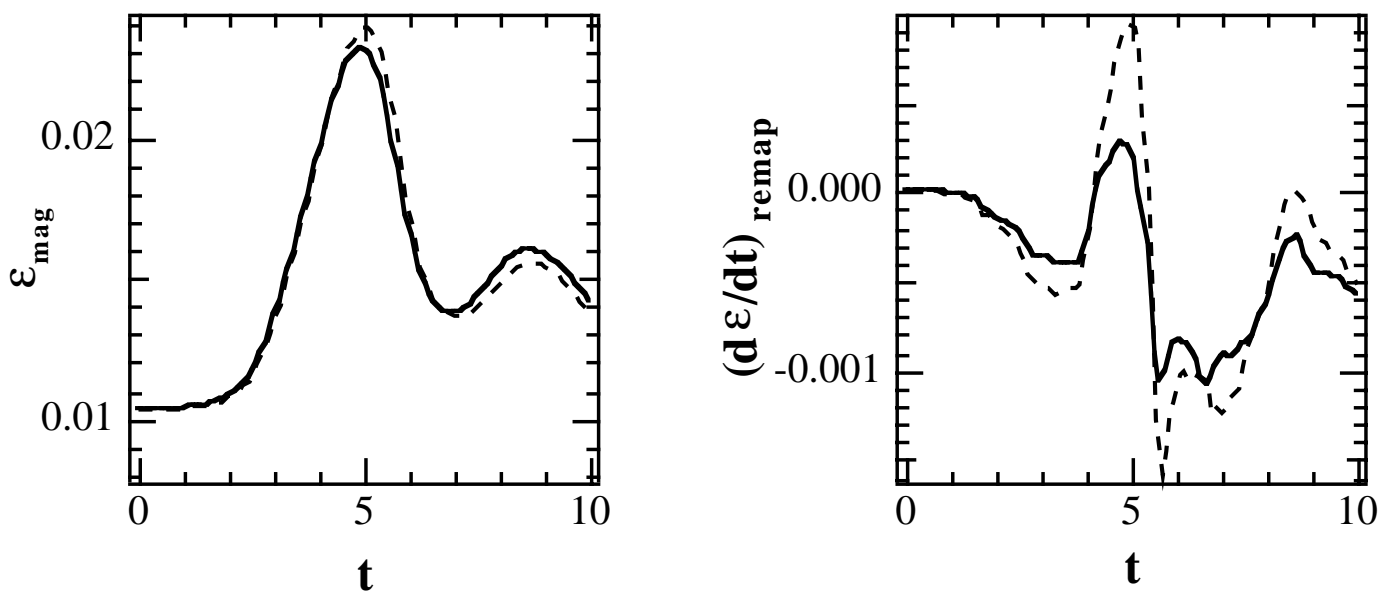

Fig. 4 Time histories from calculation presented in Fig. 3 of magnetic field energy and rate of change of magnetic field energy from remap: using second order Van Leer limited remap (solid line), and from calculation using first order remap (dashed line). Time is normalized to $\tau_{0} \equiv \mathrm{R}_{0} / \mathrm{C}_{\mathrm{s}}$ and energy to $\varepsilon_{0} \equiv \rho_{0} \mathrm{C}_{\mathrm{s}}^{2} \mathrm{~L}_{0}^{2}$. 